Filmes Biodegradáveis de Amido e Composições com Outros Materiais

\author{
Carmo, K. P.;* Paiva, J. M. F.
}

Rev. Virtual Quim., 2015, 7 (6), 2377-2386. Data de publicação na Web: 3 de outubro de 2015

http://www.uff.br/rvq

\title{
Biodegradable Films and Starch Compositions with Other Materials
}

Abstract: Due to growing concern about the environment, the use of biodegradable polymeric materials has been studied in order to minimize the environmental impact caused by synthetic polymers. Among these materials can be detached polymeric films containing corn starch, with low cost and available worldwide. This paper exhibits some characteristics of the starch and summarizes studies in which the authors present some properties of polymeric materials containing starch, especially mechanical and barrier properties, permeability to water vapor and the use of plasticizers.

Keywords: Starch; film; biodegradable polymer; properties.

\section{Resumo}

Devido à crescente preocupação com o meio ambiente, a utilização de materiais poliméricos biodegradáveis vem sendo estudada, a fim de minimizar os impactos causados por polímeros sintéticos. Dentre estes materiais podem ser destacados os filmes poliméricos contendo amido de milho, que possuem baixo custo e estão disponíveis em todo mundo. Este artigo exibe algumas características do amido e resume trabalhos nos quais os autores expõem algumas propriedades de materiais poliméricos contendo amido, principalmente, propriedades mecânicas, de barreira, permeabilidade ao vapor de água e a utilização de plastificantes.

Palavras-chave: Amido; filme; polímero biodegradável; propriedades.

* Universidade Federal de São Carlos, Campus Sorocaba, Programa de Pós-graduação em Ciência dos Materiais, CEP 18052-780, Sorocaba-SP, Brasil.

M karinapalmezani@hotmail.com

DOI: $\underline{10.5935 / 1984-6835.20150141}$ 


\title{
Filmes Biodegradáveis de Amido e Composições com Outros Materiais
}

\author{
Karina P. do Carmo, ${ }^{a, *}$ Jane Maria F. de Paiva ${ }^{a, b}$ \\ a Universidade Federal de São Carlos, Campus Sorocaba, Programa de Pós-graduação em \\ Ciência dos Materiais, CEP 18052-780, Sorocaba-SP, Brasil. \\ ${ }^{\mathrm{b}}$ Universidade Federal de São Carlos, Campus Sorocaba, Departamento de Engenharia de \\ Produção, CEP 18052-780, Sorocaba-SP, Brasil. \\ * karinapalmezani@hotmail.com
}

Recebido em 14 de abril de 2015. Aceito para publicação em 29 de setembro de 2015

\section{Introdução}

2. Fontes de Amido

3. Utilização de plastificantes

\section{Propriedades mecânicas}

5. Propriedades de barreira e permeabilidade

\section{Conclusões}

\section{Introdução}

Frequentes estudos estão sendo conduzidos para a transformação de matéria prima de fontes renováveis em embalagens biodegradáveis utilizadas para diversos fins, com o intuito de reduzir o impacto ambiental causado por polímeros sintéticos. ${ }^{1-6} \mathrm{~A}$ degradação de polímeros biodegradáveis ocorre através da ação de microrganismos tais como bactérias, fungos e algas de ocorrência natural. ${ }^{7}$

Os polímeros biodegradáveis podem ser obtidos de forma natural durante os ciclos de crescimento de organismos vivos, ou podem ser sintetizados. São provenientes de fontes variadas, dentre os quais podem ser citados os derivados de fontes microbianas, derivados da biomassa e os quimicamente sintetizados. ${ }^{8}$

Como exemplos de polímeros de fontes microbianas encontram-se os poli(hidroxialcanoatos) (PHAs), como o poli(hidroxibutirato) (PHB), o poli(hidroxivalerato) (PHV) e o poli(hidroxibutirato-co-valerato) (PHB-V). ${ }^{8}$

Dentre os polímeros derivados da biomassa, destacam-se os polissacarídeos (amidos, celulose, materiais lignocelulósicos, quitina, quitosana, pectinas) e as proteínas (caseína, zein, soja, glúten). ${ }^{8}$

Os polímeros quimicamente sintetizados podem ser produzidos a partir de monômeros oriundos de produtos de biomassa renováveis como os poli(ácido lático) (PLA), ou de monômeros de origens 
fósseis não renováveis como os poli(butileno adipato co-tereftalato) (PBAT) e as poli( $\varepsilon$ caprolactona) (PCL). ${ }^{8}$

Dentre as matérias-primas derivadas da biomassa, pode ser destacado o amido de milho, produzido mundialmente, com baixo custo e alto potencial de biodegradabilidade, podendo ser empregado em blendas ou como matriz em compósitos, dependendo da aplicação e propriedade desejada., ${ }^{5,-11}$

De acordo com o 10 Levantamento sobre a Safra Mundial de Milho 2014/15 da USDA (United States Department of Agriculture), o Brasil é o terceiro maior produtor de milho, com produção estimada em 75,0 milhões de toneladas $(\mathrm{t})$, e o segundo maior exportador, com 19,5 milhões de $t$; sendo a produção mundial prevista em 991,3 milhões de $t$ com variação de aumento de $0,23 \%$ em relação à safra de $2013 / 14 .^{12}$

\section{Fontes de Amido}

O amido é um polissacarídeo encontrado, em forma de grânulos como reserva de carboidratos em diversas espécies vegetais, tais como cereais, leguminosas e tubérculos, com composições que variam de 30 a $90 \%$ de seu peso seco, dependendo da origem vegetal em que é extraído. ${ }^{13}$

Sua estrutura é composta, principalmente, por cadeias de amilose e amilopectina, que são polímeros de glicose, possuindo funções e estruturas diferentes. A amilose possui cadeia essencialmente linear, constituída de ligações $\alpha-(1 \rightarrow 4)$ em unidades de D-glicose, e a amilopectina possui cadeia ramificada, constituída de ligações $\alpha-(1 \rightarrow 4)$, cujas ramificações encontram-se em $\alpha-(1 \rightarrow 6)$, em unidades de D-glicose. ${ }^{14}$

Dependendo da fonte vegetal de onde é extraído o amido, o percentual de amilose e de amilopectina pode variar, por exemplo, podendo o amido nativo apresentar de 20 a $35 \%$ de amilose, e o amido ceroso conter um percentual abaixo de $15 \%$ de amilose. ${ }^{15}$

$\mathrm{O}$ amido de milho na sua forma nativa pode conter a proporção de $25 \%$ a $28 \%$ de amilose e 72 a $75 \%$ de amilopectina, e existe também o amido de milho ceroso (waxy), que pode apresentar até $100 \%$ de amilopectina. ${ }^{16-18}$

Os amidos possuem caráter hidrofílico e seus filmes, geralmente, são quebradiços e apresentam baixa resistência mecânica, podendo limitar suas aplicações. ${ }^{19}$

Para a formação de filmes, a estrutura semicristalina granular do amido deve ser rompida para transformação em um material termoplástico. Tal transformação ocorre através da gelatinização, quando há a presença de excesso de água e aquecimento. Assim, ocorre uma mistura termomecânica promovendo, inicialmente, a transformação do amido em uma solução viscoelástica, dando mobilidade as cadeias até o rompimento das ligações de hidrogênio, destruindo assim sua cristalinidade. ${ }^{8,20}$

Além de serem utilizados por diversos segmentos da indústria, como a de alimentos, adesivos, agroquímica, cosmética, de papel, entre outras, o amido de milho pode ser comercializado na forma de embalagens poliméricas; e pesquisas estão sendo realizadas visando o aperfeiçoamento da produção e o desenvolvimento de compósitos, blendas e filmes poliméricos biodegradáveis. ${ }^{3,5,10,11,18,21-23}$

\section{Utilização de plastificantes}

Os plastificantes, na formação de filmes de amido, são de extrema importância, pois agem aumentando a mobilidade das cadeias poliméricas, além de auxiliar na redução da temperatura de fusão do amido, facilitando o seu processamento. Assim, ocorre melhora na flexibilidade dos filmes formados e aumento do alongamento ou da deformação, devido à redução das interações entre as cadeias do amido. ${ }^{24}$

O principal plastificante natural utilizado para produção de filmes à base de amido é a água, porém, devido ao caráter hidrofílico 
dos amidos, faz-se necessário também a utilização de outros tipos de plastificantes. ${ }^{6}$

Em vários estudos, dentre os plastificantes que possuem afinidade com os amidos podem ser citados os polióis, como o glicerol e o sorbitol. ${ }^{10,11,18,25-27}$

Segundo Mali; Grossmann; Yamashita (2010), além dos plastificantes, podem ser utilizados alguns tipos de aditivos, como pigmentos, aromatizantes, agentes antimicrobianos e antioxidantes, a fim de adicionar ou preservar algumas características desejadas para determinadas aplicações na produção de filmes de amido. ${ }^{20}$

De acordo com Zullo; lannace (2009) que analisaram os efeitos de diferentes fontes de amido (milho, batata e trigo) e diferentes tipos de plastificantes (glicerol e mistura de uréia/formamida) usados no processo de sopro, foi constatado entre as 10 diferentes composições testadas no processo, variando o tipo de amido e o percentual de plastificantes (20 e 30\%), que o filme utilizando uréia/formamida como plastificante obteve melhor desestruturação do amido e reduziu a retrogradação. Além disto, o filme de amido de milho contendo $30 \%$ de uréia/formamida apresentou aumento na deformação e alongamento na ruptura (de 0,56 $\mathrm{mm}$ para $2,12 \mathrm{~mm}$ ), tendo melhor resultado no processo de sopro quando comparado ao filme contendo glicerol. $^{28}$

Outro estudo utilizando vários tipos de plastificantes, foi realizado por Schmitt et al. (2015), ${ }^{6}$ que incorporou ao amido de trigo quatro tipos diferentes de plastificantes, dentre eles o glicerol, o sorbitol e misturas de glicerol/sorbitol e uréia/atanolamina com percentuais de $24 \%$ em peso, para formação de filmes termoplásticos por um processo de cisalhamento por extrusão. Dentre os filmes processados, a mistura contendo o plastificante ureia/etanolamina apresentou maior eficácia, devido a uma limitação no processo de retrogradação. Este processo ocorre quando as moléculas do amido gelatinizado começam a se recombinar e reagrupar, favorecendo a formação de uma estrutura mais ordenada, cristalina ocasionando a diminuição da ductilidade do material.

No caso da utilização de polióis (glicerol e sorbitol) como plastificantes, ocorreram melhorias nas propriedades mecânicas dos filmes, com o aumento da resistência à tração e do módulo de elasticidade, valores que chegaram a percentuais para o glicerol de até $346 \%$ e $1280 \%$ respectivamente, e, para as misturas de glicerol/sorbitol percentuais de até $69 \%$ e $382 \%$ respectivamente; onde cada tipo de plastificante pôde ser utilizado em diferentes aplicações, dependendo da propriedade desejada para o material em desenvolvimento. ${ }^{6}$

\section{Propriedades mecânicas}

Dentre as propriedades do amido, as mecânicas são bastante relevantes, pois são as que mais podem restringir a aplicação dos filmes poliméricos.

Em geral, as propriedades podem ser afetadas por diversos fatores e parâmetros, dentre eles podem ser destacados, o uso de plastificantes, a cristalinidade, a resistência à abrasão, resistência à tensão, e o percentual de deformação dos filmes formados.

Conforme a quantidade de plastificante utilizada na produção dos filmes de amido é aumentada, ocasiona maior mobilidade das cadeias poliméricas, elevando o percentual de deformação e, por outro lado, diminuindo os valores de tensão na ruptura. ${ }^{21,24}$

Geralmente, estruturas com maior grau de cristalinidade são mais resistentes mecanicamente, pois há um aumento na resistência ao escoamento e na dureza do material. $^{29}$

$\mathrm{Na}$ literatura podem ser encontrados vários estudos realizados com a motivação de melhorar as propriedades mecânicas dos filmes de amido de variadas fontes, 
utilizando-os em blendas poliméricas e/ou como matriz em compósitos, com a incorporação de fibras naturais, óleos essenciais, proteínas, materiais lignocelulósicos, nanopartículas, entre outros, como reforço, sempre com o intuito de preservar a biodegradabilidade dos mesmos. ${ }^{10,23,30-34}$

Estudos realizados por Dai et al. (2015), que incorporaram nanopartículas de amido de taro (Colocasia esculenta L. Schott) como agentes de reforço em filmes de amido de milho, notaram uma melhora significativa na resistência à tração, valores estes que aumentaram de 1,11 MPa para 2,87 MPa em relação aos filmes de amido de milho. ${ }^{30}$

Outros estudos utilizando nanofibras de celulose e nanocristais de celulose também obtiveram resultados satisfatórios em relação às propriedades mecânicas. ${ }^{31,32}$ De acordo com Savadekar; Mhaske (2012), a incorporação de nanofibras de celulose como reforço em matriz de amido termoplástico, ocasionou aumento de $46,1 \%$ na resistência máxima à tração do biocompósito. ${ }^{31}$

De acordo com Gilfillan et al. (2012), a adição de fibras de bagaço de cana-deaçúcar, em filmes de amido de batata e amido de milho contendo $5 \%$ em massa de fibra de bagaço, aumentou o módulo de elasticidade e a resistência a tração, além de melhoras significativas nas propriedades térmicas e uma pequena redução na absorção de água dos filmes, possivelmente, devido ao aumento da cristalinidade do material. Em posteriores estudos, Gilfillan; Moghaddam; Doherty (2014) utilizaram nanofibras de bagaço de cana-de-açúcar, com o mesmo intuito de melhorar as propriedades dos filmes poliméricos. Neste caso, utilizaram apenas o amido de batata, obtendo bons resultados com a adição de 10 a $20 \%$ em massa de fibras, tendo a resistência à tração aumentada em até 100\% (3,1-6,2 MPa) e o módulo de Young aumentado em até $300 \%(66,3-198,3 \mathrm{MPa})$. Os autores atribuíram tais melhoras às fortes interações entre o amido e as nanofibras, evidenciadas pelo aumento nos módulos de armazenamento e módulos de perda, assim como o aumento na temperatura de transição vítrea $\left(T_{\mathrm{g}}\right)$, indicando uma diminuição na mobilidade das cadeias. ${ }^{33,34}$

De acordo com Lopez et. al. (2014), filmes de amido de milho incorporados com biopolímeros de quitosana e quitina apresentaram aumento na cristalinidade, devido a algumas alterações em suas cadeias, ocasionada pela interação entre os grupos hidroxilas do amido e os grupos amino da quitosana e quitina, além de melhoras nas propriedades de resistência à tração e módulo de elasticidade, porém, houve uma diminuição no alongamento à ruptura. ${ }^{10}$

Conforme Ghasemlou et al. (2014), óleos essenciais de Zataria multiflora Boiss e Mentha pulegium incorporados a filmes de amido de milho, proporcionaram elevações consideráveis no percentual de alongamento na ruptura dos filmes poliméricos, porém, no que diz respeito aos valores de resistência à tração, estes não obtiveram alterações significativas. ${ }^{23}$

$\mathrm{Na}$ literatura são encontrados alguns trabalhos que incorporaram amidos para a formação de blendas, visando dentre outros fatores, melhorias nas propriedades e redução do custo na produção de filmes com alguns polímeros biodegradáveis, como por exemplo, o poli(hidroxibutirato) (PHB), poli(hidroxivalerato) (PHV) e o poli(hidroxibutirato-co-valerato) (PHB-V), e outros biopolímeros como os o poli(ácido lático) (PLA), poli(butileno adipato cotereftalato) (PBAT), e as poli( $\varepsilon$-caprolactona) (PCL). ${ }^{35-41}$

Lai; Don; Huang (2006) utilizaram dois diferentes tipos de amido (milho e batata), ambos com a adição do polímero poli(hidroxibutirato) (PHB) nas proporções de 1, 3, 5, 7\% em massa. Neste estudo, foi constatada uma significativa melhora na resistência à tração das blendas, conforme o percentual de PHB era aumentado, fato que foi atribuído à sua elevada rigidez e boa compatibilidade do PHB com o amido. No caso, o resultado mais satisfatório ocorreu para a blenda contendo amido de batata e $7 \%$ de $\mathrm{PHB}$, com valor máximo de 5,9 $\mathrm{MPa}$; 
porém, este valor ainda é baixo em relação à resistência à tração do PHB "puro", que é de aproximadamente $30,5 \mathrm{MPa}{ }^{36}$

No trabalho realizado por Kim; Lee; Kim (2015), o amido de milho foi agregado ao poli(butileno adipato co-tereftalato) (PBAT), para diminuir o custo do mesmo, bem como a utilização de polímeros compatibilizantes de metileno difenil diisocianato (MDI). A mistura dos polímeros PBAT/amido, diminuiu a resistência à tração, conforme foi aumentando o percentual de amido de milho $(10,20,30$ e $40 \%)$, devido à pouca interação entre as interfaces hidrofóbicas do PBAT e hidrofílicas do amido. Porém, conforme foi aumentado gradativamente $\mathrm{o}$ teor de $\mathrm{MDI}$ $(0,2-2 \%)$ ao mesmo tempo em que o percentual de amido, resultou em um aumento na resistência à tração. Neste caso, os autores atribuíram 0 aumento, possivelmente, pela melhora das interações de ligações cruzadas entre o PBAT e o amido. $^{38}$

Em um trabalho utilizando amido de mandioca, blenda de poli(butileno adipato co-tereftalato) (PBAT) e nanopartículas de argilas sepiolita, foi reportado que as nanopartículas de sepiolita são promissoras para serem utilizadas como reforço em materiais com base de amido termoplástico, pois, com a adição de apenas 3\% em massa de argila de sepiolita na matriz de amido, houve uma considerável melhora nas propriedades mecânicas, com aumento de $300 \%$ no módulo de Young e de $150 \%$ na resistência à tração, devido às fortes interações de hidrogênio entre os grupos silanol da argila sepiolita e os grupos hidroxila do amido. ${ }^{40}$

De acordo com Nuona et al. (2015), foi adicionado amido nativo cationizado ao poli(ácido lático) (PLA), nas quantidades de $400 \mathrm{mg}$ e $4 \mathrm{~g}$ respectivamente, para reduzir o custo da produção de PLA. Neste trabalho foi realizada a incorporação de óxido de grafeno como agente de compatibilização entre o PLA e o amido nativo. No caso, o amido nativo foi cationizado e depois encapsulado com o óxido de grafeno, convertendo em hidrofóbica a superfície do amido, e resultando numa forte adesão interfacial entre o amido catiônico e o PLA. Este novo método proporcionou um aumento na resistência à tração do material, pois o resultado foi de $41,4 \mathrm{MPa}$ no caso do compósito, quando comparado ao PLA "puro", cujo valor era de 36,64 MPa. ${ }^{39}$

\section{Propriedades de barreira e permeabilidade}

Uma potencial aplicação para polímeros biodegradáveis é a obtenção de filmes para embalagens de produtos de diversos tipos, como frascos para cosméticos, embalagens para adubos em grânulos ou em pó, embalagens utilizadas na agricultura para 0 plantio de mudas, embalagens comestíveis para produtos alimentícios, entre outros. ${ }^{1,4,42}$

No caso de produtos alimentícios e seu devido armazenamento, alguns fatores e propriedades específicas para utilização de embalagens poliméricas devem ser levados em consideração, tais como permeabilidade a gases e vapores de água, a fim de evitar a contaminação através da exposição a diferentes ambientes, preservando suas características e aumentando o tempo de consumo. $^{2}$

As propriedades de barreira podem ser afetadas diretamente pela presença de bolhas, fissuras, defeitos, polaridade das moléculas envolvidas, e até mesmo pelo grau de cristalinidade dos filmes poliméricos. A permeação de gases ou vapores de água se difunde através das regiões amorfas e de maior mobilidade entre as cadeias dos polímeros semicristalinos, indo do lado mais concentrado para o menos concentrado dos filmes. ${ }^{2}$

No caso dos filmes à base de amido, que possuem caráter hidrofílico, o uso de determinadas porcentagens de plastificantes, como o glicerol, contribui para o aumento da absorção de umidade, tanto do ambiente, 
quanto do local onde podem ser armazenados ou utilizados tais filmes. ${ }^{43}$

Algumas pesquisas sobre as propriedades de barreira dos filmes de amido, utilizando óleos essenciais como Zataria multiflora Boiss ou Mentha pulegium em percentuais de $1 \%$, $2 \%$ e $3 \%(v / v)$ e óleo essencial de orégano em percentuais de $0,1,0,5$ e $1 \%$ em massa, contra a ação de algumas bactérias e microrganismos, tiveram resultados satisfatórios. No caso, a adição dos mesmos na composição dos filmes, obtiveram uma redução ou até mesmo a inibição de atividades microbianas. Tais resultados, também foram alcançados na diminuição da permeabilidade aos vapores de água. ${ }^{19,23}$

No estudo de LOPEZ et al., (2014), a incorporação de quitosana e de quitina, na matriz de amido de milho, ocasionou diminuição de até $56 \%$ na permeabilidade dos filmes ao vapor de água. ${ }^{10}$

A incorporação de nanopartículas de amido de taro (Colocasia esculenta L. Schott) em filmes poliméricos de amido de milho também ocasionou diminuição significativa na permeabilidade ao vapor de água dos filmes. $^{30}$

De acordo com Gilfillan; Moghaddam; Doherty (2014), reforços utilizando nanofibras de bagaço de cana-de-açúcar em filmes a base de amido, proporcionaram redução entre 17 a $58 \%$ de absorção de umidade relativa, dependendo da quantidade em massa de bagaço utilizado. ${ }^{34}$

Segundo Brandelero; Grossmann; Yamashita (2013), blendas de amido de mandioca e poli(butileno adipato cotereftalato) (PBAT) com adição de 0,5\% de óleo de soja, se apresentaram menos hidrofílicos, pois o óleo de soja ocasionou aumento das frações hidrofóbicas nos filmes elaborados, acarretando na redução dos coeficientes de solubilidade e difusão, proporcionando às blendas elaboradas, maior barreira aos vapores de água. ${ }^{37}$

\section{Conclusões}

Através das literaturas citadas, os amidos, mesmo sendo provenientes de fontes renováveis e biodegradáveis, apresentam desvantagens quando comparados aos polímeros sintéticos devido aos baixos valores das propriedades mecânicas, a grande afinidade de absorção de água, e dificuldades em se estabelecer parâmetros para o processamento dos filmes, principalmente em larga escala.

Assim foram encontrados diversos trabalhos com o intuito principal de melhorar propriedades mecânicas de filmes contendo amido e outros polímeros naturais biodegradáveis, utilizando combinações destes com outros materiais, desde óleos vegetais até alguns tipos de argilas, obtendose blendas poliméricas e materiais compósitos.

\section{Agradecimentos}

A CAPES pela bolsa de Mestrado de K. P. do Carmo. Aos docentes da disciplina de Projetos de Dissertação do Programa de Pósgraduação em Ciência dos Materiais (PPGCM)/UFSCar Campus Sorocaba. Ao Prof. Dr. Walter Ruggeri Waldman pelo grande incentivo à elaboração deste trabalho.

\section{Referências Bibliográficas}

${ }^{1}$ Olivato, J. B.; Mali, S.; Grossmann, M. V. E. Efeito de embalagem biodegradável de amido no armazenamento de queijo processado. Semina Ciências Agrárias 2006, 27, 81. [CrossRef]

${ }^{2}$ Siracusa, V. Food Packaging Permeability Behaviour: A Report. International Journal of Polymer Science 2012, 302029. [CrossRef]

${ }^{3}$ Heydari, A.; Alemzadeh, I.; Vossoughi, M. Functional properties of biodegradable corn starch nanocomposites for food packaging 
applications. Materials and Design 2013, 50, 954. [CrossRef]

${ }^{4}$ Bilck, A. P.; Olivato, J. B.; Yamashita, F.; de Souza, J. R. P. Biodegradable Bags for the Production of Plant Seedlings. Polímeros: Ciência e Tecnologia 2014, 24, 547. [Link]

${ }^{5}$ Lopez, O. V.; Castillo, L. A.; Garcia, M. A.; Villar, M. A.; Barbosa, S. E. Food packaging bags based on thermoplastic corn starch reinforced with talc nanopalticles. Food Hydrocolloids 2015, 43, 18. [CrossRef]

${ }^{6}$ Schmitt, H.; Guidez, A.; Prashantha, K.; Soulestin, J.; Lacrampe, M. F.; Krawczak, P. Studies on the effect of storage time and plasticizers on the structural variations in thermoplastic starch. Carbohydrate Polymers 2015, 115, 364. [CrossRef]

${ }^{7}$ Rosa, D. S.; Chui, Q. S. H.; Pantano Filho, R.; Agnelli, J. A. M. Evaluation of PHB, PHB-V and $P C L$ biodegradation in composted soil. Polímeros: Ciência e Tecnologia 2002, 12, 311. [CrossRef]

${ }^{8}$ Avérous, L.; Pollet, E. Em Environmental Silicate Nano-Biocomposites, Green Energy and Technology; Avérous, L.; Pollet, E., eds.; Springer Verlag: London, 2012, cap. 2.

${ }^{9}$ Guimarães, J. L.; Wypych, F.; Saul, C. K.; Ramos, L. P.; Satyanarayana, K. G. Studies of the processing and characterization of corn starch and is composites with banana and sugarcane fibers from Brazil. Carbohydrate Polymers 2010, 80, 130. [CrossRef]

10 Lopez, O.; Garcia, M. A., Villar, M. A.; Gentili, A.; Rodrigues, M. S.; Albertengo, L. Thermo-compression of biodegradable thermoplastic corn starch films containing chitin and chitosa. LWT - Food Science and Technology 2014, 57, 106. [CrossRef]

${ }^{11}$ Slavutsky, A. M.; Bertuzzi, M. A. Water barrier properties of starch films reinforced with cellulose nanocrystals obtained from sugarcane bagasse. Carbohydrate Polymers 2014, 110, 53. [CrossRef]

12 Sítio da Federação das Indústrias do Estado de São Paulo. Disponível em: $<$ http://www.fiesp.com.br/indices-pesquisas- e-publicacoes/safra-mundial-de-milho-2/>.

Acesso em: 21 fevereiro 2014.

${ }^{13}$ Denardin, C. C.; da Silva, L. P. Starch granules structure and its regards with physicochemical properties. Ciência Rural 2009, 39, 945. [CrossRef]

${ }^{14}$ Ellis, R. P.; Cochrane, M. P.; Dale, M. F. B.; Duffus, C. M.; Lynn, A.; Morrison, I. M.; Prentice, R. D. M.; Swanston, J. S.; Tiller, S. A. Starch production and industrial use. Journal of the Science of Food and Agriculture 1998, 77, 289. [CrossRef]

${ }^{15}$ Tester, R. F.; Karkalas, J.; Qi, X. Starch composition, fine structure and architecture. Journal of Cereal Science 2004, 39, 151. [CrossRef]

${ }^{16}$ Chaudhary, A. L.; Torley, P. J.; Halley, P. J.; McCaffery, N.; Chaudhary, D. S. Amylose content and chemical modification effects on thermoplastic starch from maize - Processing and characterisation using conventional polymer equipment. Carbohydrate Polymers 2009, 78, 917. [CrossRef]

${ }^{17}$ Wu, R. L.; Wang, X. L.; Li, F.; Li, H. Z.; Wang, Y. Z. Green composite films prepared from cellulose, starch and lignin in roomtemperature ionic liquid. Bioresource Technology 2009, 100, 2569. [CrossRef]

${ }^{18}$ Campagner, M. R.; Moris, V. A. S.; Pitombo, L. M.; do Carmo, J. B.; de Paiva, J. M. F. Polymeric Films Based on Starch and lignosulfonates: Preparation, Properties and Evaluation of Biodegradation. Polímeros: Ciência e Tecnologia 2014, 24, 740. [Link]

${ }^{19}$ Pelissari, F. M.; Grossmann, M. V. E.; Yamashita, F.; Pineda, E. A. G. Antimicrobial, Mechanical, and Barrier Properties of Cassava Starch-Chitosan Films Incorporated with Oregano Essential Oil. Journal of Agricultural and Food Chemistry 2009, 57, 7499. [CrossRef]

${ }^{20}$ Mali, S.; Grossmann, M. V. E.; Yamashita, F. Filmes de amido: produção, propriedades e potencial de utilização. Semina Ciências Agrárias 2010, 31, 137. [CrossRef]

${ }^{21}$ Corradini, E.; Lotti, C.; Medeiros, E. S. de; Carvalho, A. J. F.; Curvelo, A. A. S.; Mattoso, L. H. C. Estudo comparativo de amidos termoplásticos derivados do milho com 
diferentes teores de amilose. Polímeros: Ciência e Tecnologia 2005, 15, 268. [CrossRef]

${ }^{22}$ Kaith, B. S.; Jindal, R.; Jana, A. K.; Maiti, M. Development of corn starch based green composites reinforced with Saccharum spontaneum L fiber and graft copolymers Evaluation of thermal, physico-chemical and mechanical properties. Bioresource Technology 2010, 101, 6843. [CrossRef]

${ }^{23}$ Ghasemlou, M.; Aliheidari, N.; Fahmi, R.; Shojaee-Aliabadi, S.; Keshavarz, B.; Cran, M. J.; Khaksar, R. Physical, mechanical and barrier properties of corn starch films incorporated with plant essential oils. Carbohydrate Polymers 2013, 98, 1117. [CrossRef]

${ }^{24}$ Galdeano, M. C.; Mali, S.; Grossmann, M. V. E.; Yamashita, F.; Garcia, M. A. Effects of plasticizers on the properties of oat starch films. Materials Science and Engineering: $C$ 2009, 29, 532. [CrossRef]

${ }^{25}$ Mueller, C. M. O.; Laurindo, J. B.; Yamashita, F. Effect of cellulose fibers addition on the mechanical properties and water vapor barrier of starch-based films. Food Hydrocolloids 2009, 23, 1328. [CrossRef] ${ }^{26}$ Lopez, O. V.; Zaritzky, N. E.; Grossmann, M. V. E.; Garcia, M. A. Acetylated and native corn starch blend films produced by blown extrusion. Journal of Food Engineering 2013, 116, 286. [CrossRef]

${ }^{27}$ Prachayawarakorn, J.; Chaiwatyothin, S.; Mueangta, S.; Hanchana, A. Effect of jute and kapok fibers on properties of thermoplastic cassava starch composites. Materials and Design 2013, 47, 309. [CrossRef]

${ }^{28}$ Zullo, R.; lannace, S. The effects of different starch sources and plasticizers on film blowing of thermoplastic starch: Correlation among process, elongational properties and macromolecular structure. Carbohydrate Polymers 2009, 77, 376. [CrossRef]

${ }^{29}$ Canevarolo, S. V.; Bernardo, F. O. C.; Hincapie, J. A. Z.; Elias, M. B.; Silva, J. Structural Characterization of Bi-oriented Blown Films of Polypropylene/Polyethylene Blends. AIP Conference Proceedings 2014, 1593, 650. [CrossRef]
${ }^{30}$ Dai, L.; Qiu, C.; Xiong, L.; Sun, Q. Characterisation of corn starch-based films reinforced with taro starch nanoparticles. Food Chemistry 2015, 174, 82. [CrossRef]

${ }^{31}$ Savadekar, N. R.; Mhaske, S. T. Synthesis of nano cellulose fibers and effect on thermoplastics starch based films. Carbohydrate Polymers 2012, 89, 146. [CrossRef]

${ }^{32}$ Alves, J. S.; dos Reis, K. C.; Menezes, E. G. T.; Pereira, F. V.; Pereira, J. Effect of cellulose nanocrystals and gelatin in corn starch plasticized films. Carbohydrate Polymers 2015, 115, 215. [CrossRef]

${ }^{33}$ Gilfillan, W. N.; Nguyen, D. M. T.; Sopade, P. A.; Doherty, W. O. S. Preparation and characterisation of composites from starch and sugar cane fibre. Industrial Crops and Products 2012, 40, 45. [CrossRef]

${ }^{34}$ Gilfillan, W. N.; Moghaddam, L.; Doherty, W. O. S. Preparation and characterization of composites from starch with sugarcane bagasse nanofibres. Cellulose 2014, 21, 2695. [CrossRef]

${ }^{35}$ Franchetti, S. M. M.; Marconato, J. C. Biodegradable polymers - a partial way for decreasing the amount of plastic waste. Química Nova 2006, 29, 811. [CrossRef]

${ }^{36}$ Lai, S. M.; Don, T. M.; Huang, Y. C. Preparation and properties of biodegradable thermoplastic starch/poly(hydroxy butyrate) blends. Journal of Applied Polymer Science 2006, 100, 2371. [CrossRef]

${ }^{37}$ Brandelero, R. P. H.; Grossmann, M. V.; Yamashita, F. Hydrophilicity of Starch and Poly(Butylene Adipate-Co-Terephthalate) (Pbat) Films Containing Tween 80 and Soybean Oil. Polímeros: Ciência e Tecnologia 2013, 23, 270. [CrossRef]

${ }^{38}$ Kim, J.-H.; Lee, J. C.; Kim, G.-H. Study on poly(butylene adipate-coterephthalate)/starch composites with polymeric methylenediphenyl diisocyanate. Journal of Applied Polymer Science 2015, 132, 41884. [CrossRef]

${ }^{39}$ Nuona, A.; Li, X.; Zhu, X.; Xiao, Y.; Che, J. Starch/polylactide sustainable composites: 
Interface tailoring with graphene oxide. Composites Part A: Applied Scence and Manufacturing 2015, 69, 247. [CrossRef]

${ }^{40}$ Olivato, J. B.; Marini, J.; Pollet, E.; Yamashita, F.; Grossmann, M. V. E.; Avérous, L. Elaboration, morphology and properties of starch/polyester nano-biocomposites based on sepiolite clay. Carbohydrate Polymers 2015, 118, 250. [CrossRef]

${ }^{41}$ Yu, Y.; Cheng, Y.; Ren, J.; Cao, E.; Fu, X.; Guo, W. Plasticizing effect of poly(ethylene glycol)s with different molecular weights in poly(lactic acid)/starch blends. Journal of
Applied Polymer Science 2015, 132, 41808. [CrossRef]

${ }^{42}$ Silva-Pereira, M. C.; Teixeira, J. A.; PereiraJunior, V. A.; Stefani, R. Chitosan/corn starch blend films with extract from Brassica oleraceae (red cabbage) as a visual indicator of fish deterioration. LWT - Food Science and Technology 2015, 61, 258. [CrossRef]

${ }^{43}$ Corradini, E.; Teixeira, E. M.; Agnelli, J. A. M.; Mattoso, L. H. C. Amido termoplástico. Disponível em: <http://www.infoteca.cnptia.embrapa.br/ha ndle/doc/30796>. Acesso em: 18 junho 2014. 